\title{
Análise cienciométrica da integração energética em biorrefinarias de cana-de-açúcar
}

\section{Gabrielly Mylena Benedetti Tonial*, Paulo Roberto Paraíso e Luiz Mário de Matos Jorge}

Universidade Estadual de Maringá. Departamento de Engenharia Química. Bloco D90. Av. Colombo, 5790. Jardim Universitário. Maringá-PR, Brasil. (CEP 87020-680).*E-mail: gabriellybene@gmail.com.

Resumo. Com o intuito de quantificar os estudos envolvendo integração energética, bem como fazer um levantamento específico ao que se refere à biorrefinarias de cana-de-açúcar, realizou-se um estudo cienciométrico detalhado relacionado à integração energética nessas usinas. 0 levantamento das informações se deu pela base de dados de Web of Science, utilizando os termos "Energy Integration AND Biorefinery", "Heat Integration AND Biorefinery", "Energy Integration AND Sugarcane" e "Heat Integration AND Sugarcane" como palavraschave, no período de 2007 a 2019. Dentre as instituições mais relevantes na pesquisa as que mais publicaram foram a Chalmers University of Technology (Suiça) e a Universidade Estadual de Campinas (Brasil), enquanto considerando as 133 referências estudadas, a "Energy" foi o periódico que rendeu mais publicações e citações.

Palavras-chave: Análise bibliométrica; Integração de calor; Publicações; Web of Science.

Abstract. Bibliometric analysis of energy integration in sugarcane biorefineries. In order to quantify the studies involving energy integration, as well as to make a specific survey regarding the sugarcane biorefineries, a detailed scientometric study was carried out related to the energy integration in these biorefineries. The information was collected using the Web of Science database, using the terms "Energy integration and Biorefinery", "Heat Integration AND Biorefinery", "Energy Integration AND Sugarcane" and "Heat Integration AND Sugarcane" as keywords, in the period from 2007 to 2019. Among the most relevant institutions in the research, the most published were Chalmers University of Technology (Switzerland) and the Universidade Estadual de Campinas (Brazil), while considering the 133 references studied, "Energy" was the periodical that yielded more publications and citations.

Keywords: Bibliometric analysis; Heat integration; Publications; Web of Science.

Recebido

25/05/2021

Aceito

$15 / 12 / 2021$

Publicado

$31 / 12 / 2021$

Acesso aberto

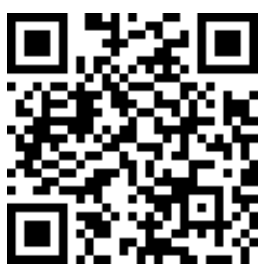

ORCID

(1) 0000-0002-2729-8332

Gabrielly Mylena

Benedetti Tonial

(D) 0000-0003-3178-8104 Paulo Roberto Paraíso

(D) 0000-0003-1869-1957

Luiz Mário de Matos

Jorge

ISSN 2359-1412/RBGAS-2021-0057/2021/8/20/22/1621

Rev. Bras. Gest. Amb. Sustent.

http://revista.ecogestaobrasil.net 


\section{Introdução}

Biorrefinarias são conceituadas como um dos modelos industriais que reaproveitam os resíduos gerados (biomassa) para a transformação em produtos com valor agregado, tais como bioquímicos, biocombustíveis, bionenergia e biomateriais. (Santos et al., 2013). Naturalmente, as usinas sucroalcooleiras são consideradas como biorrefinarias, nas quais pode-se produzir o açúcar, o etanol (biocombustível) e como subproduto a energia elétrica, fornecida, principalmente, pela biomassa de cana-de-açúcar (bagaço) (Rambo et al., 2015; Cortes-Rodríguez et al., 2018; Oliveira et al., 2018). De acordo com Brito et al. (2015), essa produção de energia pode gerar autossuficiência nas indústrias, assim como, pode ser uma fonte extra de renda com a comercialização do seu excedente.

A integração energética em biorrefinarias de cana-de-açúcar possibilita a redução no consumo de utilidades quentes e frias (vapor e água), por meio de uma rede de trocadores de calor. A demanda do menor consumo de vapor é capaz de gerar uma "sobra" do bagaço da cana-de-açúcar, no qual a sua reutilização pode variar para a produção de etanol de segunda geração e/ou eletricidade, dependendo da demanda (Cortes-Rodríguez et al., 2018; Oliveira et al., 2018).

Inúmeros estudos têm sido publicados na área de otimização de processos com ênfase em integração energética em diversas indústrias químicas. Com o intuito de quantificar esses estudos, bem como fazer um levantamento específico aos que se referem a biorrefinarias de cana-de-açúcar, realizou-se um estudo cienciométrico.

A análise cienciométrica é um estudo que demonstra a evolução de produções científicas num determinado período de tempo, envolvendo qualquer área específica, em qualquer campo da ciência (Mingers e Leydesdorff, 2015). Esse estudo é amplamente utilizado como método para avaliar e examinar o desenvolvimento de uma pesquisa, com base nos esforços de pesquisadores, Instituições, países e até Periódicos em uma determinada área de pesquisa (Konur, 2012; Yonoff et al., 2019).

Diante do exposto, o presente estudo teve por objetivo realizar o levantamento de dados, por meio da análise cienciométrica, referente à integração energética em biorrefinarias de cana-de-açúcar, até o ano de 2019.

\section{Materiais e métodos}

Para o levantamento de dados de pesquisas científicas, para a análise cienciométrica, utilizou-se a base de dados de artigos científicos do Institute for Scientific Information Web of Science (ISI WoS), que é considerado, de acordo com Van Leeuwen (2006) e Leydesdorff (2016), uma das mais importantes e confiáveis fonte de dados para esse tipo de análise.

0 acesso ao WoS foi feito por meio do CAFE, na plataforma Portal de Periódicos CAPES/MEC, vinculado à Universidade Estadual de Maringá (UEM). A pesquisa foi gerada empregando-se os termos "Energy Integration AND Biorefinery", "Heat Integration AND Biorefinery", "Energy Integration AND Sugarcane" e "Heat integration AND Sugarcane" como palavras-chave, a fim de se obter uma maior restrição nos resultados. Considerou-se todas as produções bibliográficas relacionadas às palavras-chave mencionadas, até o ano de 2019.

Utilizou-se as abas "Análise de Resultados" e "Relatório de Citações" disponibilizadas no WoS. Com os dados obtidos, foram gerados os gráficos e as tabelas para a apresentação dos resultados. 


\section{Resultados e discussão}

A distribuição dos tipos de documento identificados pelo WoS foi analisada para as pesquisas realizadas com todas as palavras-chave, dos quais foram encontrados três tipos de documentos, entre os quais, artigos científicos, artigos publicados em anais de congressos (proceedings paper) e artigos de revisão (review). 0 documento no modelo de artigo científico foi o mais utilizado, considerando as quatro palavras-chave empregadas na busca, sendo 76\% para "Energy Integration AND Biorefinery" e para "Heat Integration AND Biorefinery". Percentuais maiores de $88 \%$ e $89 \%$ foram encontrados, respectivamente, pelo uso das palavras-chave "Heat Integration AND Sugarcane" e "Energy Integration AND Sugarcane".

Para todos os termos utilizados como palavras-chave, as áreas de pesquisa que se sobressaíram foram referentes à Engenharia Química eà área de Energia e Combustível, perfazendo o maior número de publicações e citações, segundo o banco de dados do WoS.

\section{Número de publicações}

Dos termos aplicados como palavras-chave, surgiu em 2007 a primeira publicação referente à "Heat Integration AND Biorefinery", sendo que as demais começaram a ser publicadas no ano de 2009. Com a interpretação desses dados, pode-se inferir que a otimização de processos na área de integração energética em biorefinarias trata-se de um assunto recente.

Os resultados referentes ao número de publicações no período de 2007 a 2019 com a utilização dos termos para palavras-chave mencionadas anteriormente, estão apresentados na Figura 1.

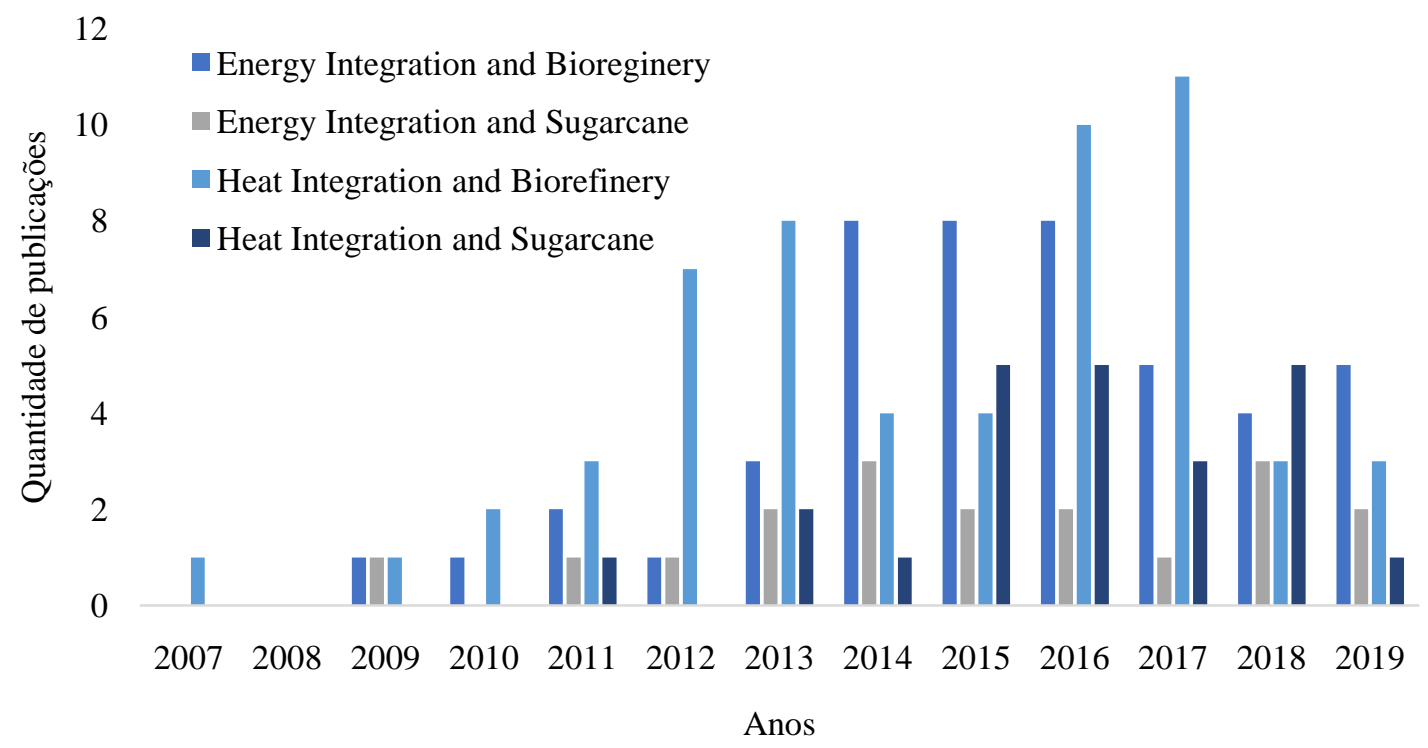

Figura 1. Quantificação de publicações no período de 2007 a 2019 para os termos "Energy Integration AND Biorefinery", "Energy Integration AND Sugarcane", "Heat Integration AND Biorefinery" e "Heat Integration AND Sugarcane".

Pelo levantamento de dados no $W o S$, considerando a área de estudo proposta, no ano de 2008 não foram encontradas publicações relacionadas aos termos de busca. A partir do ano de 2013, essas pesquisas apresentaram crescimento, havendo maior número de publicações, com destaque para os anos de 2016 e 2017. 
Dos termos aplicados para a realização da pesquisa, "Energy Integration AND Sugarcane" foi o que apresentou menor número de publicações (18) durante o período da pesquisa. Por outro lado, o termo "Heat Integration AND Biorefinery", foi o que apresentou o maior número de publicações, totalizando 57.

Considerando a pouca quantidade de artigos publicados para cada um dos termos utilizados como palavras-chave, optou-se por agrupar ambos os termos referentes à "biorefinery" e "sugarcane", a fim de se obter apenas duas vertentes de pesquisa para dar seguimento ao estudo.

\section{Distribuição de publicações por países e instituições (Universidades)}

No levantamento de produções bibliográficas por Instituições, considerou-se como corte o mínimo de quatropublicações no período de 2009 a 2019, utilizando-se como palavras-chave "Energy Integration AND Biorefinery" e "Heat Integration AND Biorefinery", totalizando 69 publicações. A relação da produção científica por instituição é apresentada na Tabela 1.

Tabela 1. Número de publicações por Instituições para os termos "Energy Integration AND Biorefinery"e"Heat Integration AND Biorefinery" como palavras-chave.

\begin{tabular}{|l|c|c|}
\hline Instituição & País & Número de publicações \\
\hline Chalmers University of Technology & Sweden & 19 \\
\hline National Technical University of Athens & Greece & 8 \\
\hline Universidad Nacional de Colombia & Colombia & 8 \\
\hline Carnegie Mellon University & USA & 5 \\
\hline Universidade Federal de São Carlos & Brazil & 5 \\
\hline Ecole Polytechnique Federale de Lausenne & Switzerland & 4 \\
\hline Universidade Estadual de Campinas & Brazil & 4 \\
\hline University of Manchester & England & 4 \\
\hline University of Nottingham Malaysia & Malaysia & 4 \\
\hline University of Salamanca & Spain & 4 \\
\hline University of Waikato & New Zealand & 4 \\
\hline Total & - & 69 \\
\hline
\end{tabular}

A Chalmers University of Technology (Sweden) contribuiu com aproximadamente $28 \%$ do total de publicações. Neste ranking duas universidades brasileiras se destacam, em 5ํ lugar, a Universidade Federal de São Carlos, e em 7ํㅡ lugar, a Universidade Estadual de Campinas, totalizando cerca de $13 \%$ da produção total.

A grande maioria das pesquisas realizadas pela Chalmers University of Technology (Sweden) leva em consideração o estudo de biorrefinarias de papel e celulose. Por outro lado, as pesquisas brasileiras são majoritariamente focadas em biorrefinarias de cana-de-açúcar. 0 foco de estudo de cada país está relacionado, possivelmente, com os recursos econômicos disponíveis em cada nação. A exemplo disso pode-se citar o Brasil como maior produtor mundial de cana-de-açúcar (CONAB, 2019).

Na Tabela 2 estão relacionadas as Instituições que mais publicaram no período de 2007 a 2019 quando utilizado para a busca os termos "Energy Integration AND Sugarcane" e "Heat Integration AND Sugarcane". Nesse caso, considerou-se para corte o número mínimo de duas publicações por Instituição, sendo 45 o total de produção científica para o período. 
Tabela 2. Número de publicações por Instituições para os termos "Energy Integration AND Sugarcane" e "Heat Integration AND Sugarcane" como palavras-chave.

\begin{tabular}{|l|c|c|}
\hline Instituição & País & Número de publicações \\
\hline Universidade Estadual de Campinas & Brazil & 12 \\
\hline Universidade Federal do ABC & Brazil & 7 \\
\hline Universidad Nacional de Colombia & Colombia & 5 \\
\hline Ecole Polytechnique Federale de Lausanne & Switzerland & 4 \\
\hline Universidade Federal de São Carlos & Brazil & 4 \\
\hline IFP Energies Nouvelles & France & 3 \\
\hline Universidade Federal de Lavras & Brazil & 2 \\
\hline Universidade Federal do Rio de Janeiro & Brazil & 2 \\
\hline Stellenbosch University & South Africa & 45 \\
\hline Total & - & \\
\hline
\end{tabular}

Dentre as instituições que mais publicaram neste período, encontra-se, em primeiro, a Universidade Estadual de Campinas (Brasil), com aproximadamente $27 \%$ do total de publicações.

Em comparação aos dados apresentados na Tabela 1, observa-se que Instituições brasileiras se destacam na produção científica referente à biorrefinaria de cana-de-açúcar, totalizando aproximadamente $62 \%$ do total de publicações neste período. A alta produção brasileira neste assunto deve-se a grande quantidade de usinas sucroalcooleiras existentes no país.

\section{Padrões de publicação: títulos de fonte (periódicos)}

Como resultado da união dos termos "Energy Integration AND Biorefinery" e "Heat Integration AND Biorefinery" foram encontrados 96 artigos publicados (excluindo os artigos repetidos) em torno de 52 revistas diferentes. Dessa maneira, para a apresentação dos periódicos levou-se em consideração o mínimo de quatro publicações para compor os dados apresentados na Tabela 3. Essa tabela apresenta também o total de citações referente a cada uma dessas revistas.

Tabela 3. Número de publicações e citações por periódico para os termos "Energy Integration AND Biorefinery" e "Heat Integration AND Biorefinery" como palavras-chave.

\begin{tabular}{|l|c|c|}
\hline Periódico & Número de publicações & Total de citações \\
\hline Bioresource Technology & 9 & 215 \\
\hline Energy & 7 & 88 \\
\hline Chemical Engineering Research\& Design & 6 & 116 \\
\hline Applied Energy & 5 & 69 \\
\hline Computers \& Chemical Engineering & 5 & 127 \\
\hline Applied Thermal Engineering & 4 & 45 \\
\hline Biochemical Engineering Journal & 4 & 42 \\
\hline Biofuels Bioproducts Biorefining Biofpr & 4 & 27 \\
\hline Biomass \& Bioenergy & 4 & 120 \\
\hline
\end{tabular}

Os nove periódicos apresentados na Tabela 3 representam $50 \%$ de todas as publicações do período de 2009 a 2019, totalizando 48 produções. Dessas produções, o PeriódicoBioresource Technology foi responsável por aproximadamente 19\% deste total 
de publicações. Além disso, as publicações dessa mesma revista geraram o maior percentual de citações, totalizando aproximadamente 25\%. As citações de cada Periódico/Ano são mostradas na Figura 2.

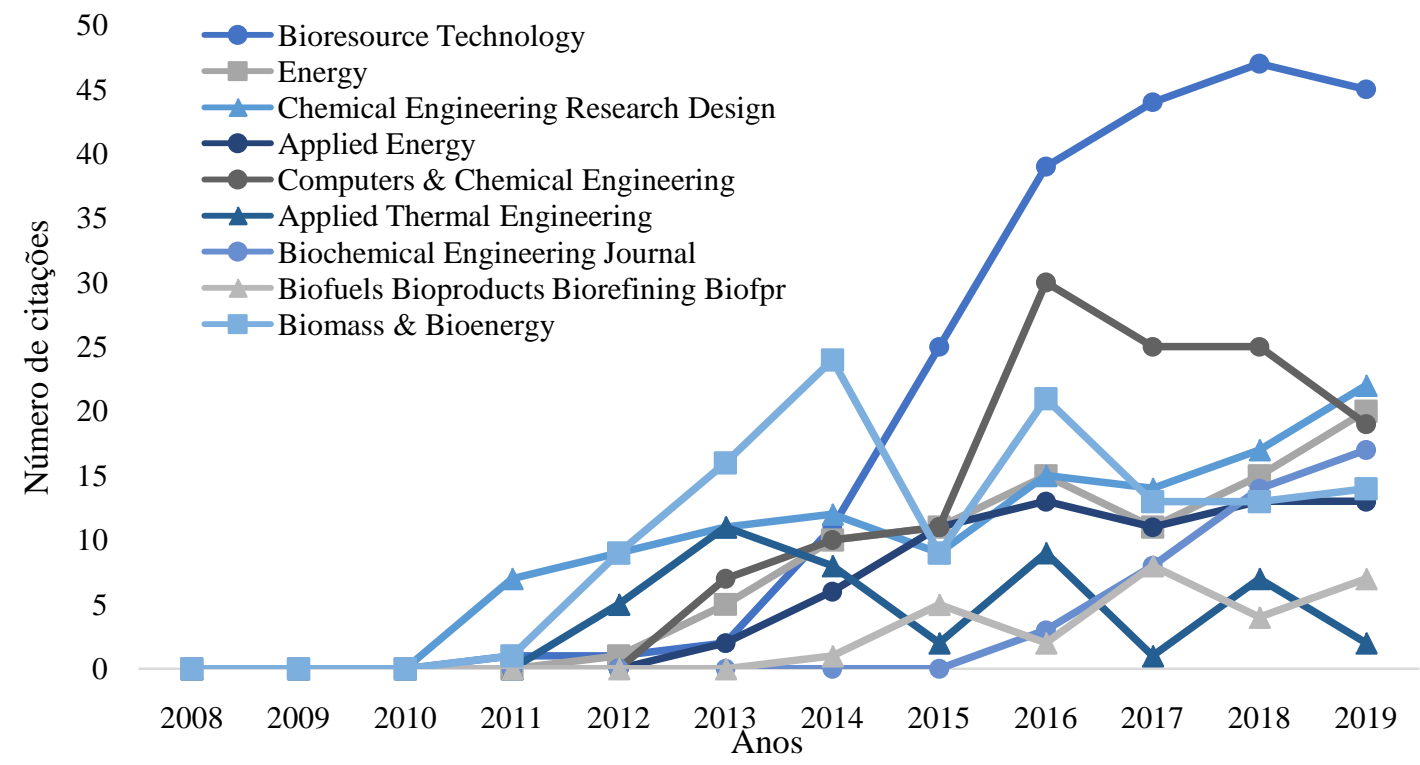

Figura 1. Número de citações por periódico/ano para os termos "Energy Integration AND Biorefinery" e "Heat Integration AND Biorefinery" como palavras-chave.

Da relação de periódicos apresentados, a revista Bioresourse Technology mostrou o número de citações crescente de 2013 a 2018, com declínio de citações no ano de 2019. Em contrapartida, a revista Chemical Engineering Research Design com menor número de citações, com relação a Bioresourse Technology, mostrou ordem crescente no período de 2010 a 2019. Da mesma forma, a revista Biochemical Engineering Journal apesar de apresentar crescimento no número de citações apenas a partir do ano de 2015, também se manteve crescente até 2019. Contudo, para as demais revistas, o número de citações apresentou variações ao longo do período.

A busca dos termos "Energy Integration AND Sugarcane" e "Heat Integration AND Sugarcane" na plataforma WoS resultou em 37 artigos publicados (excluindo os artigos repetidos) em 24 diferentes revistas. Considerou-se, para esse levantamento de dados, o mínimo de duas publicações por periódico, os quais são apresentados na Tabela 4, na qual também é apresentado o total de citações referente a cada um desses Periódicos.

Tabela 4. Número de publicações e citações por periódico para os termos "Energy Integration AND Sugarcane" e "Heat Integration AND Sugarcane" como palavras-chave.

\begin{tabular}{|l|c|c|}
\hline Periódico & $\begin{array}{c}\text { Número de } \\
\text { Publicações }\end{array}$ & $\begin{array}{c}\text { Total de } \\
\text { citações }\end{array}$ \\
\hline Energy & 7 & 188 \\
\hline Chemical Engineering Research \& Design & 4 & 89 \\
\hline Bioresource Technology & 3 & 43 \\
\hline Biomass \& Bioenergy & 2 & 38 \\
\hline Applied Energy & 2 & 16 \\
\hline
\end{tabular}


Durante o período de 2007 a 2019, as cinco revistas apresentadas na Tabela 4 somaram o total de 18 publicações, o que representa aproximadamente $49 \%$ dessas 37 produções científicas. A revista Energy é a principal fonte de publicações, perfazendo aproximadamente 39\% do total de produção apresentado na tabela. Os sete manuscritos apresentados na revista geraram aproximadamente $50 \%$ do total das citações contidas na Tabela 4. A quantidade de citações referenciadas por ano a partir de cada uma das dessas cinco revistas são mostrados na Figura 3.

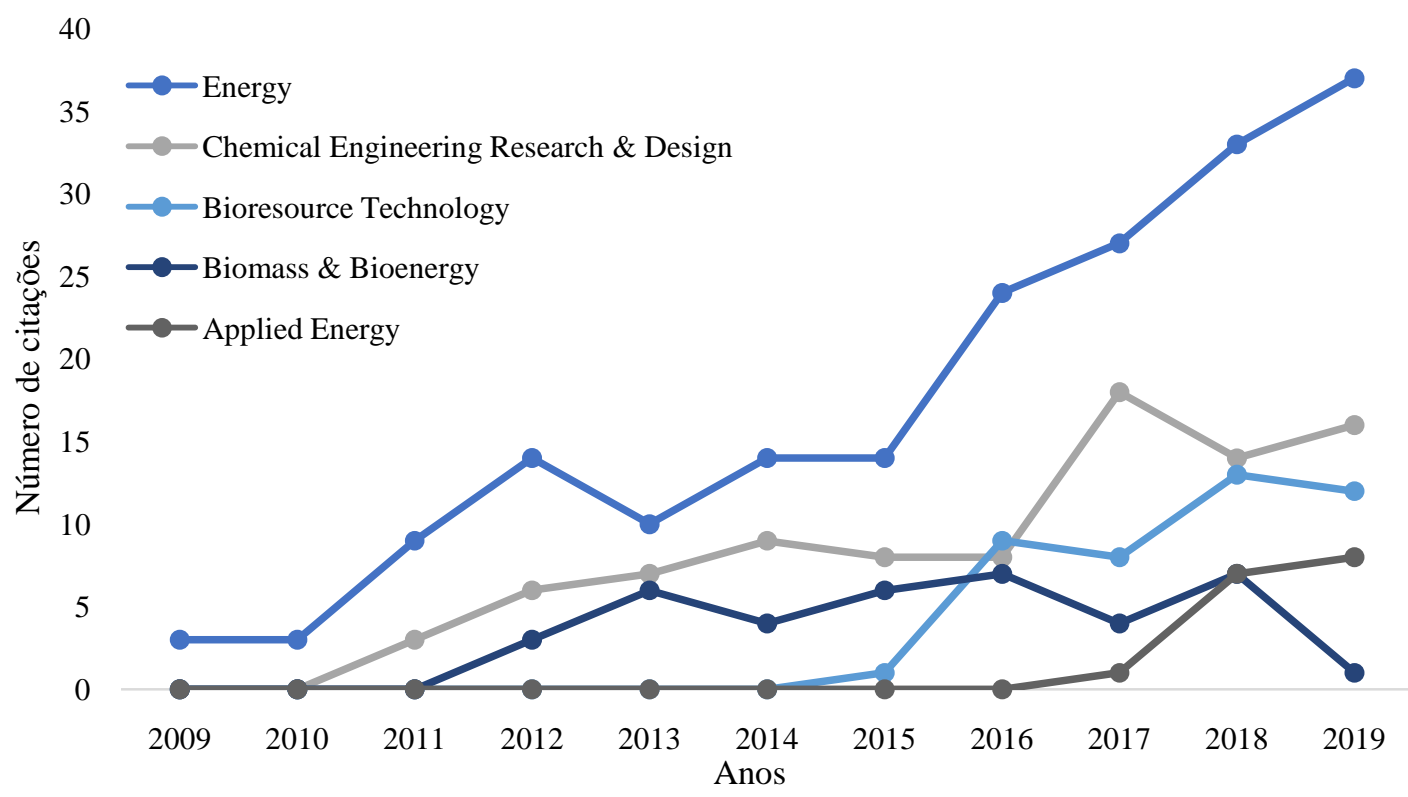

Figura 2. Número de citações por periódico/ano para os termos "Energy Integration AND Sugarcane" e "Heat Integration AND Sugarcane"como palavras-chave.

Dentre as revistas apresentadas (Figura 3), o Periódico Energy foi o primeiro a apresentar citações (2009), obtendo crescimento consecutivo a partir de 2015. Por outro lado, a revista Applied Energy começou apresentar resultados de referências somente em 2017. Contrariamente, as demais revistas apresentaram redução no número de citações nos últimos dois anos.

\section{Ênfase da pesquisa: palavras-chave do autor}

A avaliação das palavras-chave dos autores no presente estudo indica que 296 palavras-chave do autor foram usadas de 2007 a 2019. Dessas, apenas 18 palavras-chave (6\%) foram utilizadas mais de quatro vezes, as quais são apresentadas na Tabela 5.

Das 18 palavras-chave apresentadas, "Biorefinery" foi referenciada cerca de $20 \%$ do total de aparições (Tabela 5). 0 alto percentual de aparição deve-se principalmente ao fato de ser uma das palavras utilizadas nos dois dos termos de busca, os quais resultaram em 96 artigos (Tabela 1). Ao desconsiderar as palavras-chave "Biorefinery", "Energy Integration", "Heat Integration" e "Sugarcane", as quais compuseram os termos de busca, a palavra-chave "Process Integration" foi a mais referenciada, com aproximadamente $10 \%$ das citações. 
Tabela 5. Palavras-chave mais citadas pelos autores nas produções científicas, no período de 2007 a 2019, para todos os quatro termos usados na pesquisa.

\begin{tabular}{|l|c|}
\hline Palavras-chave do autor & Número de aparições \\
\hline Biorefinery & 35 \\
\hline Heat integration & 25 \\
\hline Process integration & 18 \\
\hline Energy efficiency & 11 \\
\hline Ethanol & 11 \\
\hline Pinch analysis & 10 \\
\hline Energy integration & 9 \\
\hline Bioethanol & 9 \\
\hline Process design & 7 \\
\hline Gasification & 6 \\
\hline Kraft pulp mill & 6 \\
\hline Life cycle assessment & 5 \\
\hline Bioprocess design & 4 \\
\hline Economic analysis & 4 \\
\hline Economic assessment & 4 \\
\hline Sugarcane & 4 \\
\hline Sugarcane bagasse & 4 \\
\hline Xylitol & 4 \\
\hline
\end{tabular}

Pode-se dizer que as palavras-chave de autores, listadas na Tabela 5, estão relacionadas aos seguintes aspectos de otimização de processos (heat integration, process integration, energy efficiency, pinch analysis, energy integration, process design, bioprocess design), biomassa e biocombustível (bioethanol, gasification, sugarcane bagasse, xylitol), biorrefinarias (biorefinery, ethanol, kraft pulp mil, sugarcane) e métodos de avaliação (life cycle assessment, economic analysis e economic assessment). Isso comprova que aproximadamente $48 \%$ das palavras-chave de autores estão na área de otimização de processos, o que justifica a sua preferência de estudo dentro do tema abordado.

Dentre os artigos encontrados durante a pesquisa, percebeu-se que diferentes focos de estudo são abordados. Para a área de otimização de processos, o estudo apresentado por Oliveira et al. (2016), elaborado no Brasil, realizou a integração energética, utilizando a análise Pinch, em uma biorrefinaria de cana-de-açúcar que produz etanol de primeira e segunda geração, assim como a bioeletrecidade.

Ainda no Brasil, no ano de 2019, Ortiz et al. (2019), apresentaram um estudo referente ao design de processo e avaliação de um sistema de produção de etanol à base de cana-de-açúcar. Este estudo combinou a otimização de processos por integração de massa e calor, a fim de melhorar a eficiência de produção.

Outro estudo utilizando cana-de-açúcar, expressa a simulação um novo conceito de biorrefinaria, o qual foi desenvolvido por Albarelliet al.(2018). Esse conceito tem por finalidade acrescentar ao processo a extração de cera de cana-de-açúcar, gerada a partir do resíduo de torta de filtro, o que pode ser atraente em termos econômicos com a venda desse extrato.

Cortes-Rodriguez etal. (2018) realizaram a integração energética num sistema de evaporação de efeito múltiplo para a concentração da vinhaça num processo de produção de etanol por cana-de-açúcar e a simulação de um sistema de cogeração de energia, seguido de uma avalição econômica. 
Na Suécia, a partir do estudo de Lundberg et al. (2014), foi possível obter autoeficiência em termos de vapor na produção de celulose dissolvida, com exportação de eletricidade, lignina e hemicelulose aumentando o nível de integração de calor do moinho.

Nakashimaet al. (2019), utilizaram resíduos de biorrefinarias da cana-de-açúcar com utilização de digestão aeróbica e gaseificação para geração de biocombustível e eletricidade, com a utilização da integração energética. Mountraki et al. (2017) também utilizaram resíduos agrícolas e florestais (biomassa lignocelulósica), porém para produção de xilitol, com aplicação de integração de dois processos (biotecnológico e catalítico), realizando-se também a integração energética nos processos.

\section{Conclusão}

Ao todo foram encontradas 133 produções científicas no WoS, baseada nos termos "Energy Integration AND Biorefinery", "Heat Integration AND Biorefinery", "Energy Integration AND Sugarcane" e "Heat Integration AND Sugarcane", no período de 2007 a 2019.

A instituição que mais publicou,quando se tratando dos termos "Energy Integration AND Biorefinery", "Heat Integration AND Biorefinery", foi a Chalmers University of Technology (Suiça), enquanto para os termos"Energy Integration AND Sugarcane" e "Heat Integration AND Sugarcane", a Universidade Estadual de Campinas (Brasil) foi a que mais publicou.

Os três principais periódicos em número de publicações e citações foram "Energy", "Bioresource Technology" e "Chemical Engineering Research \& Design".

As palavras-chave de autor que mais apareceram nos resumos dos artigos encontrados foram "Biorefinery", "Heat Integration" e "Process Integration".

Por fim, concluiu-se que a pesquisa relacionada a integração energética em biorrefinarias de cana-de-açúcar é recente e o seu desenvolvimento se mostra crescente nos últimos anos.

\section{Conflito de interesses}

Os autores declaram não haver conflito de interesses.

\section{Referências}

Albarelli, Q.; Santos, D. T.; Meireles, M. A. A. Thermo-economic evaluation of a new approach to extract sugarcane wax integrated to a first and second generation biorefinery. Biomass and Bioenergy, v. 119, p. 69-74, 2018. https://doi.org/10.1016/j.biombioe. 2018.09.018

Brito, C. S. B.; Oliveira, D. M.; Nascimento, G. P.; Pessoa, F. S.; Silva, D. Produção de energia elétrica a partir da biomassa de cana-de-açúcar. Anuário de Produções Acadêmicocientíficas dos Discentes da Faculdade Araguaia, v. 4, p. 121-127, 2015.

CONAB - Companhia Nacional de Abastecimento. Acompanhamento da safra brasileira de cana-de-açúcar: Safra 2020/2021. Brasília: CONAB, v. 7. n. 1, 2020. Disponível em: <https://www.conab.gov.br/info-agro/safras/cana/boletim-da-safra-de-cana-de-acucar>. Acesso em: 06 jun. 2020. 
Cortes-Rodríguez, E. F.; Fukushima, N. A.; Palacios-Bereche, R.; Ensinas, A. V.; Nebra, S. A. Vinasse concentration and juice evaporation system integrated to the conventional ethanol production process from sugarcane: Heat integration and impacts in cogeneration system. Renewable Energy, v. 115, p. 474-488. 2018. https://doi.org/10.1016/j.renene. 2017.08.036

Konur, 0. The evaluation of the global research on the education: A scientometric approach. Procedia: Social and Behavioral Sciences, v. 47, p.1363-1367, 2012. https://doi.org/10.1016/j.sbspro.2012.06.827

Leydesdorff, L.; Wouters, P.; Bornmann, L. Professional and citizen bibliometrics: Complementarities and ambivalences in the development and use of indicators-a state-ofthe-art report. Scientometrics, v. 109, p. 2129-2150, 2016. https://doi.org/10.1007/ s11192-016-2150-8

Lundberg, V.; Bood, J.; Nilsson, L.; Axelsson, E.; Berntsson, T.; Svensson E. Converting a kraft pulp mill into a multi-product biorefinery: Techno-economic analysis of a case mill. Clean Technologies Environmental Policy, v. 16, p. 1411-1422, 2014. https://doi.org/ 10.1007/s10098-014-0741-8

Nakashima, R. N.; Florez-Orrego, D.; Oliveira Junior, S. Integrated anaerobic digestion and gasification processes for upgrade of ethanol biorefinery residues. Journal of Power Technologies, v. 99, p.104-114, 2019. Disponível em: <https://papers.itc.pw.edu.pl/ index.php/JPT/article/view/1475>. Acesso em: 29 maio 2020.

Mingers, J.; Leydesdorff, L. A review of theory and practice in scientometrics. European Journal of Operational Research, v. 246, p. 1-19, 2015. https://doi.org/10.1016/ j.ejor.2015.04.002

Mountraki, A. D.; Koutsospyros, K. R.; Mlayah, B. B.; Kokossis, A. C. Selection of biorefinery routes: The case of xylitol and its integration with an organosolv process. Waste and Biomass Valorization, v. 8, p. 2283-2300, 2017. https://doi.org/10.1007/s12649-0169814-8

Oliveira, C. M.; Cruz, A. J. G.; Costa, C. B. B. Improving second generation bioethanol production in sugarcane biorefineries through energy integration. Applied Thermal Engineering, v. 109, p.819-827, 2016. https://doi.org/10.1016/j.applthermaleng. 2014.11.016

Oliveira, C. M.; Pavão, L. V.; Ravagnani, M. A. S. S.; Cruz, A. J. G.; Costa, C. B. B. Process integration of a multiperiod sugarcane biorefinery. Applied Energy, v. 213, p. 520-539, 2018. https://doi.org/10.1016/j.apenergy.2017.11.020

Ortiz, P. A. S.; Maciel, R.; Posada, J. Mass and heat integration in ethanol production mills for enhanced process efficiency and exergy-based renewability performance. Process, v. 7, n. 670, p. 1-31, 2019. https://doi.org/10.3390/pr7100670

Rambo, M. K. D.; Alexandre, G. P.; Rambo, M. C. D.; Alves, A. R.; Garcia, W. T.; Baruque, E. Characterization of biomasses from the north and northeast regions of Brazil for processes in biorefineries. Food Science and Technology, v. 35, p.605-6011, 2015. https://doi.org/10.1590/1678-457X.6704

Santos, F.; Colodette, J.; Queiroz, J. H. Bioenergia e biorrefinaria: cana-de-açúcar e espécies florestais. Viçosa: Editora UFV, 2013.

Van Leeuwen, T. The application of bibliometric analyses in the evaluation ofsocial science research. Who benefits from it, and why it is still feasible. Scientometrics, v. 66, p. 133154. 2006. https://doi.org/10.1007/s11192-006-0010-7 
Yonoff, R. E.; Ochoa, G. V.; Cardenas-Escorcia, Y.; Silva-Ortega, J. I.; Meriño-Stand, L. Research trends in proton exchange membrane fuel cells during 2008-2018: A bibliometric analysis. Heliyon, v. 5, p. 1-7, 2019. https://doi.org/10.1016/j.heliyon.2019. e01724

\footnotetext{
(CC) Informação da Licença: Este é um artigo Open Access distribuído sob os termos da Licença Creative Commons Attribution, que permite uso irrestrito, distribuição e reprodução em qualquer meio, desde que a obra original seja devidamente citada.
} 